\title{
Polypropylene Fibers Crimped by Asymmetrical Quenching
}

\author{
By Tatsuki Matsuo, Member, TMSJ \\ Katata Research Center, Toyobo Co., Ltd., Otsu, Shiga Pref. \\ Based on the Journal of the Textile Machinery Society of Japan, Transactions, Vol. 27, No. 11, T 177-182 (1974)
}

\begin{abstract}
Polypropylene crimped fibers can be produced by asymmetrical quenching under some spinning conditions. This paper deals with those spinning conditions and crimping mechanisms to get the following results:

1. The spinning conditions necessary and sufficient for providing polypropylene fibers with crimps are:

i) Extruded molten filaments should be asymmetrically quenched by cross air flow.

ii) The density of undrawn fibers thus obtained should be within a certain optimum range.

2. This optimum range of density varies with the linear density of fibers as shown in Fig. 8 .

3. The windward side of a filament is located in the inner side of a helical crimp.

4. The diameter of a helical crimp formed by asymmetrical quenching is calculated by a theoretical equation derived in the previous report ${ }^{[22]}$.

5. The reason why crimpability is the largest at such an optimum density is analysed.

6. It is experimentally and theoretically shown that the effect of the linear density on the helical crimp diameter is smaller in asymmetrical quenching than in bicomponent spinning.

7. Crimpability can be intensified with an increase of quenching air velocity and by spinning hollow fibers.
\end{abstract}

\section{Introduction}

It has so far been well known that good crimped fibers can be produced by asymmetrically condensating the viscose liquid extrudated from nozzle orifices ${ }^{[1]}$. There have also a lot of literatures which deal with crimped fibers obtained by asymmetrically quenching the thread lines in melt spinning. ${ }^{[2-3]}$ Methods for producing crimped fibers can be classified as follows: One is mechanical crimping such as false-twisting or stuffing box crimping. Another is to utilize the latent crimpability grown in spinning. As to the latter, there are two spinning methods: bicomponent and asymmetrical condensating. For bicomponent spinning, a certain special apparatus is required to conjugate two components of dopes. On the contrary, no special apparatus, is necessary for asymmetrical quenching. Therefore, asymmetrical quenching has a great advantage over bicomponent spinning from the view point of investment cost.

Because threads are generally cooled by cross air flow in melt spinning, they are more or less asymmetrically quenched as to their fiber axes. But as is well known, most undrawn fibers so far obtained by melt spinning have never latent crimpability. It is thought that, in addition to asymmetrical quenching, some other special conditions are necessary for providing undrawn fibers with latent crimpability.
Among fibers commercially produced by melt spinning, polypropylene (PP) fibers seem to be most easily crimped by asymmetrical quenching. The fact that there have been many patents ${ }^{[14-19]}$ concerning PP fibers crimped by asymmetrical quenching may suggest the importance of the crimped PP fibers. Nevertheless, little work has been done on the precise crimping conditions and crimping mechanism of these fibers.

With regard to producing crimped fibers by bicomponent spinning or asymmetrical quenching, crimp formation is usually carried out by heating drawn fibers in a relaxed state. This paper concerns mainly in the melt spinning process of PP fibers, and investigates the precise conditions for impregnating them with latent crimpability. The mechanism of latent crimp formation is also discussed.

\section{Experiments}

\subsection{Polymer used}

Material: iso-tactic polypropylene (Moplene FB), Intrinsic viscosity (see 2.4): 1.55

Tacticity (residue extracted by $n$-heptane): $95 \%$

2.2 Standard spinning conditions

Spinning machine: A test machine whose capacity is $1 \mathrm{~kg} / \mathrm{hr}$

Take-up speed: $600 \mathrm{~m} / \mathrm{min}$ 
Quenching conditions: Air carefully regulated at $20^{\circ} \mathrm{C}$ is blown at $0.8 \mathrm{~m} / \mathrm{sec}$ across thread lines at $20 \mathrm{~mm}$ below the spinneret.

Spinneret: The number of holes having the diameter of $0.8 \mathrm{~mm}$ is 10 or 20 .

2.3 Standard drawing and heating conditions

Drawing: Yarns are drawn at $25^{\circ} \mathrm{C}$ between two rolls with the drawing ratio of 3 .

Heat treatment: After reeled, drawn yarns are heated in a relaxed state for 10 mins in a circulated air bath at $120^{\circ} \mathrm{C}$.

Unless the conditions are specially mentioned, all the experiment is carried out according to the standard conditions mentioned above.

2.4 Measurements on fiber properties

(1) Undrawn fibers

Intrinsic viscosity is measured in $135^{\circ} \mathrm{C}$ tetraline solution.

Density is measured by means of density gradient tubes in which the liquid is composed of isopropylalcohol and water at $30^{\circ} \mathrm{C}$.

Latent crimpability is evaluated by the diameter of the helical crimp obtained by the following procedures: An undrawn fiber is drawn three times as long as the original in $20^{\circ} \mathrm{C}$ air at the extension rate of $20 \% / \mathrm{sec}$. Then it is quickly relaxed in $20^{\circ} \mathrm{C}$ air.

(2) Drawn or heat-treated fibers

Shrinkage percentage: The linear density " $b$ " of the drawn fibers is measured after conditioning at $20^{\circ} \mathrm{C}$ for $24 \mathrm{hrs}$. in a tensionless state. Its linear density " $\mathrm{c}$ " is measured after additional heating for $20 \mathrm{mins}$ in a relaxed state in an air bath at $120^{\circ} \mathrm{C}$.

Shrinkage percentage $=[\{$ (bor c $)-a\} /($ bor c $)] \times 100(\%)$, where $a=$ (the linear density of the undrawn fiber)/ (drawing ratio)

Crimp percentage and number of crimps: Those of the heat-treated fibers are measured according to JIS 1074 and 1090 .

It has been confirmed that there is a close corelationship between the latent crimpability of undrawn fibers and the crimp percentage of drawn and heat-treated fibers.

\section{Experimental results and discussions}

3.1 Basic conditions for producing latent crimpability Spinning tests were carried out at various spinneret temperatures, while the linear density of undrawn fibers was kept at 5 denier and the distance between the spinneret and the upper end of quenching air was varied to 20,45 and $140 \mathrm{~mm}$. The experimental result of the relationship between the intrinsic viscosity (with reference to the spinneret temperature) and the crimp percentage is shown in Fig. 1. The crimp percentage takes fairly sharp peaks at an optimum spinneret temperature at each distance be-

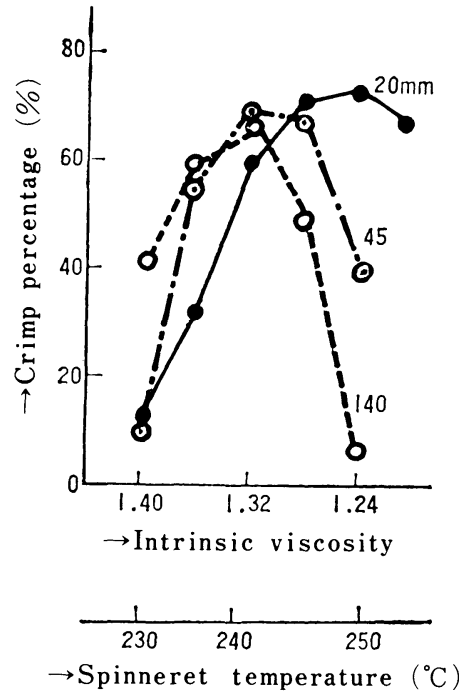

Fig. 1 Changes in crimpability with spinneret temperature (Figures on the curve show the distances between the spinneret and the upper end of quenching air)

tween the spinneret and the upper end of quenching air. Therefore this optimum spinneret temperature can not be singularly determined, but varies with the change of the quenching conditions. But a singular tendency appears as shown in Fig. 2 by plotting the data against the density of undrawn fibers. Such a tendency seems to be unchanged even if other conditions change excepting the linear density of undrawn fibers and the kind of polypropylene resin used.

Two kinds of undrawn fibers were obtained at the standard conditions by blowing cool air across threadlines in the mutually opposite two directions as shown in Fig. 3. (The linear density of the single filament in this case was

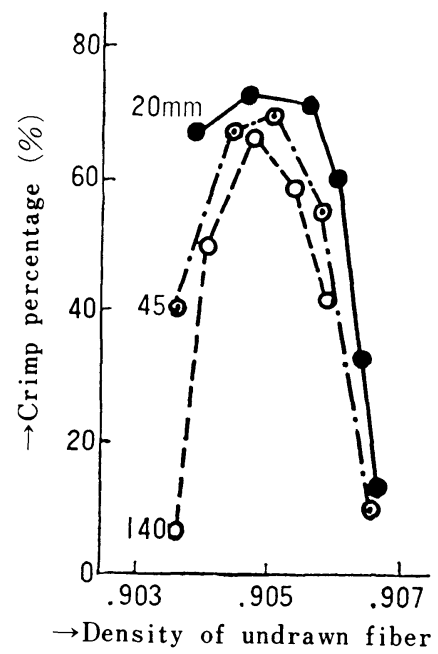

Fig. 2 Changes in crimpability with density of undrawn fiber (Figures on the curves show the distances between the spinneret and the upper end of quenching air) 


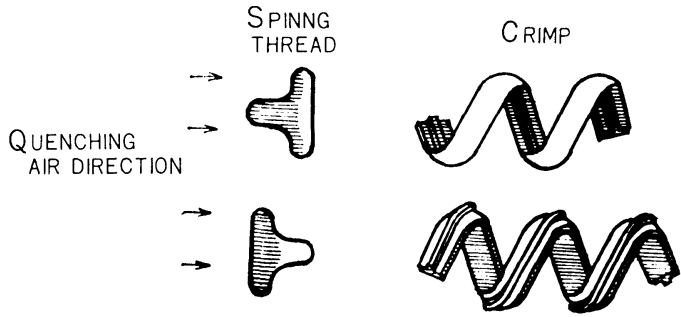

Fig. 3 The relationship between quencing air direction and in or outside of a helical crimp

30 den.) The shapes of helical crimps obtained by drawing and then heat-treating these fibers in a relaxed state are shown in Fig. 3. It shows that the windward side of the filament is located at the inner sides of helical crimps.

\subsection{Crimping mechanism}

The relationship between the density of undrawn fibers and the thermal shrinkage of fibers produced by drawing these fibers was experimentally obtained as shown in Fig. 4. The thermal shrinkage percentage increases with the increase of the density (i.e. crystallinity) of undrawn fibers. The curves takes a sigmate form and its inflextion point is near the density of $0.904\left(\mathrm{~g} / \mathrm{cm}^{3}\right)$. The experimental data

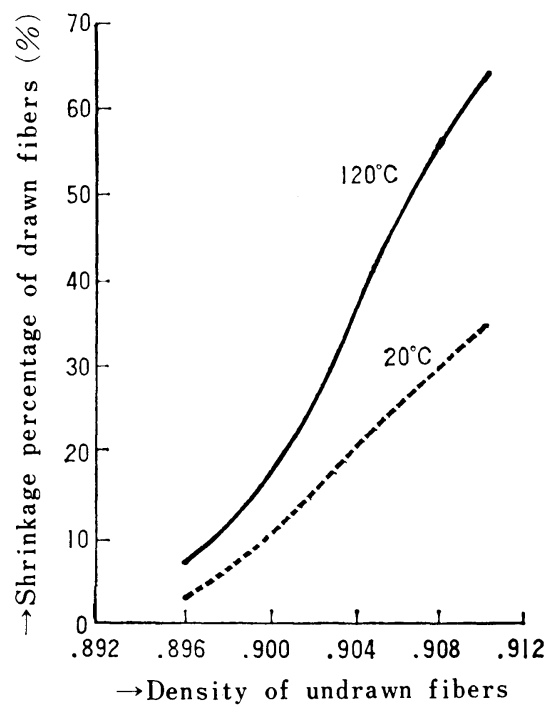

Fig. 4 The relationship between shrinkage percentage of drawn fibers and density of undrawn fibers (Figures show heat-treatment temperature) described in Table 4 of the previous report ${ }^{[21]}$ show that the density of undrawn fibers is always higher in the windward side than in the leeward side of a filament. This fact and the relationship shown in Fig. 4 may naturally explain why the windward side is located in the inner sides of helical crimps.

Now let us discuss the radius of a helical crimp. The theoretical equation for the radius of a helical crimp was derived in the previous report ${ }^{[22]}$ as follows:

$$
\frac{1}{R}=-\frac{A_{t}}{Z} \frac{\int_{c} \frac{y}{1-S} d A}{\int_{c} \frac{1}{1-S} d A} \ldots \ldots \ldots \ldots \ldots \ldots(1)
$$

where $R$; the radius of a helical crimp

$A t$; the cross-sectional area of a filament

$Z$; the cross-sectional 2 nd order moment of a filament relating to its neutral line

$S$; shrinkage

$y$; the coordinate in the radial direction of a helical crimp, as shown in Fig. 5

$\int_{c}$; surface integral for all the cross-sectional area As shown in Fig. 2, the crimp percentage reached the maximum at the density of $0.905\left(\mathrm{~g} / \mathrm{cm}^{3}\right)$ with undrawn fibers, where the single filament denier is 5 denier. At this point, the latent crimpability took the value of $0.3 \mathrm{~mm}$. Further, the crimped fiber whose single filament denier is 2.8 and the number of crimps is 40 was obtained by drawing this undrawn fiber three times as long as the original and then heating it in a relaxed state at $120^{\circ} \mathrm{C}$ for $10 \mathrm{~min}$.

As previously reported, ${ }^{[21]}$ the temperature within the filament asymmetrically quenched takes such a profile as shown in Fig. 6. Further, this temperature profile may directly influence on the crystallinity profile within an undrawn filament. On the basis of these facts, calculations were attempted to get the radius of a helical crimp by means of eq. (1). In this case, the shrinkage within the drawn filament is assumed to distribute as shown in Table 1, by making use of the relationship of Fig. 4. Calculated results are entered in Table 1 in comparisons with experimental ones. Experimental datum No. 1 corresponds to the radius of latent crimpability. Experimental datum No. 2 was derived from the number of crimps of heat-relaxed fibers by means of the following equation;

Radius of latent crimpability $=25 /\{\pi \times($ crimp number $\}$ (mm)

Table 1 Relationship between cross-sectional shrinkage distributions and diameters of helical coil

\begin{tabular}{|c|c|c|c|c|c|c|c|c|c|c|c|}
\hline \multirow{2}{*}{ No. } & \multicolumn{9}{|c|}{ Cross-sectional shrinkage distributions ( $\%$ ) } & \multicolumn{2}{|c|}{$\begin{array}{l}\text { Dia. of helical } \\
\text { coil }(\mathrm{mm})\end{array}$} \\
\hline & I & II & III & IV & V & VI & VII & VIII & IX & Cal. & Exp. \\
\hline 1 & 16.2 & 17.3 & 18.7 & 20.1 & 21.5 & 22.9 & 24.3 & 25.7 & 27.0 & 0.43 & 0.33 \\
\hline 2 & 30.0 & 32.7 & 35.5 & 38.3 & 45.1 & 43.8 & 46.6 & 49.3 & 52.0 & 0.19 & 0.20 \\
\hline
\end{tabular}




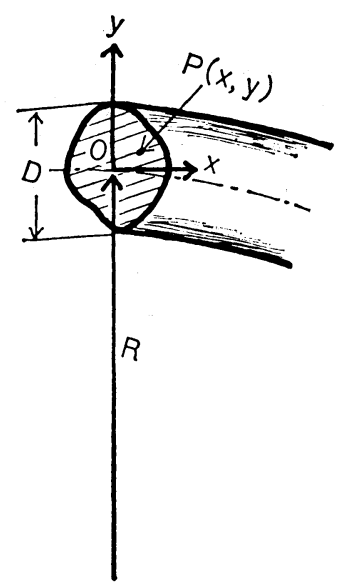

Fig. 5 Coordinates for calculating the radius of a helical crimp

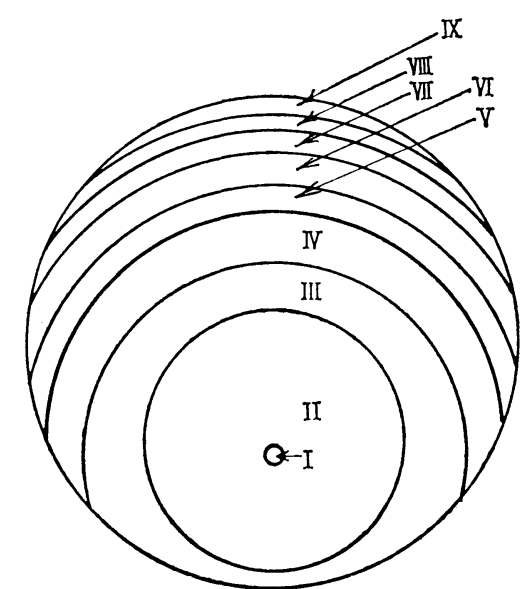

Fig. 6 Temerature profile within a filament asymmetrically quenched

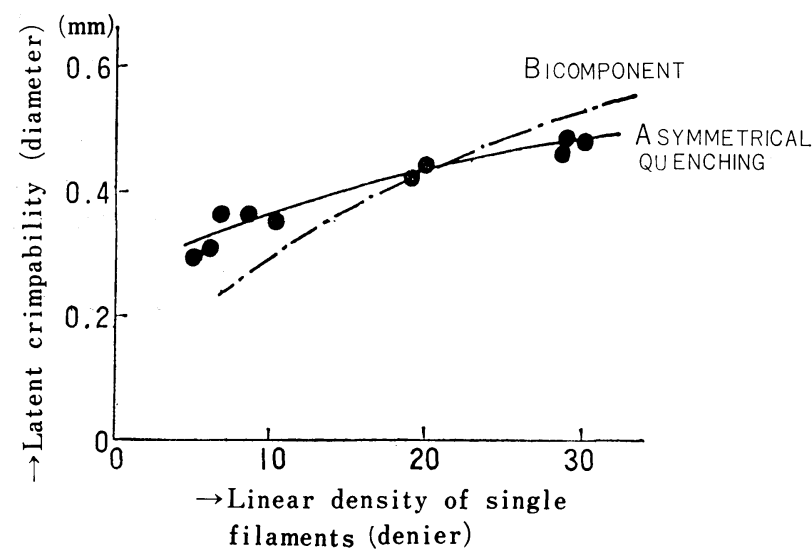

Fig. 7 Changes in the maximum crimpability with linear density of single filaments
Experimental datum No. 2 agrees fairly well with calculated one. But there is a little difference between experimental datum of No. 1 and calculated ones. This may be originated from the errors in the assumed distribution of shrinkage entered in No. 2 column of Table 1.

As previously reported ${ }^{[22]}$, the helical crimp radius of bicomponent fibers can be given by the following equation, where suffixes show each component:

$$
\begin{gathered}
\frac{1}{R}=\frac{E_{1} E_{2}\left(A_{2} Z_{1}-A_{1} Z_{2}\right)}{E_{1} I_{1}+E_{2} I_{2}} \times \\
\frac{S_{1}-S_{2}}{\left(1-S_{1}\right) A_{2} E_{2}+\left(1-S_{2}\right) A_{1} E_{1}}
\end{gathered}
$$

where $E$; Young's modulus

$S$; degree of shrinkage

$A$; cross-sectional area

$Z$; cross-sectional 2nd order moment

The next approximate equation can easily be deduced from eq. (2):

$R \propto{\text { (the linear density of a single filament })^{1 / 2}}^{1 / 2}$

On the contrary, the precise radius of fibers crimped by asymmetrical quenching should be calculated directly by means of eq. (1). For brevity, if the shrinkage distribution is approximated by the following equation;

$$
S \fallingdotseq-K(y / D)+S_{o}
$$

eq. (1) can be simplified as follows:

$$
1 / R \doteqdot K / D
$$

where $D$ is the diameter of a filament. The coefficient $K$ in eq. (3) is the shrinkage gradient in the direction from windward to leeward across the fiber axis. According to the previous report, ${ }^{[22]} K$ depends mainly on Biot number ( $=h D / k p$, where $h$ is the heat transfer coefficient, $k p$ the thermal conductivity of polymer), and increases with the increase of Biot number. Therefore it can be supposed that $K$ increases with the increase in the linear density of single filaments, as presumed from eq. (8) in the previous report. ${ }^{[22]}$ From these reasons, it is forecast that the crimp radius of the fiber produced by asymmetrical quenching does not so strongly depend on the linear density of single filaments as that of bicomponent fibers. The latent crimpability of the undrawn fiber produced under the optimum condition is plotted against the linear density of each single filament in Fig. 7. The theoretical curve for bicomponent fibers obtained from eq. (2) is also drawn in Fig. 7. It supports the above described forecast. That is, the crimp produced by asymmetrical quenching does not become so fine even if the linear density of a single filament decreases. On the contrary, it does not become so coarse with the increase of the linear density. These facts may suggest that the fiber crimped by asymmetrical quenching is more useful in the application fields of comparatively larger linear density.

As above mentioned, the crimpability of PP fibers produced by asymmetrical quenching takes its maximum value 


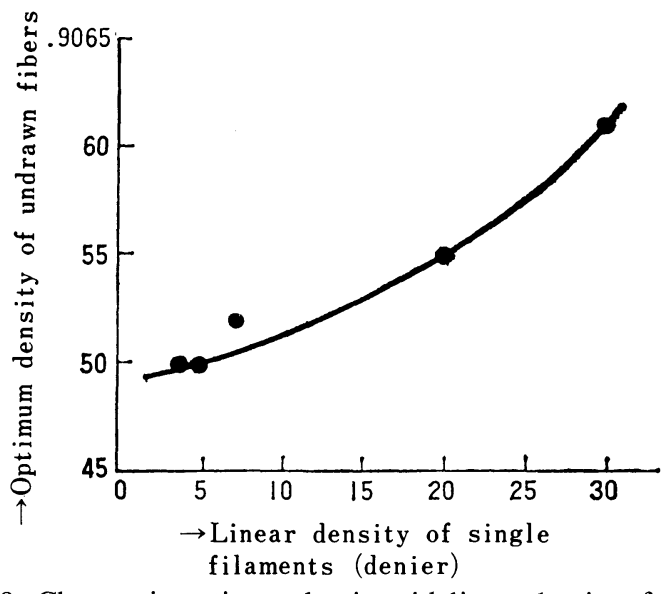

Fig. 8 Changes in optimum density with linear density of single filaments

at a certain optimum density of undrawn fibers. This seems to be caused by the fact that the shrinkage of drawn fibers increases sigmately with the increase in the density of undrawn fibers as shown in Fig. 4. The sloep of this curve is proportional to the coefficient $K$ in eq. (4). The point at which the slope becomes the maximum, namely the inflextion point of this curve should corresponds to the optimum point for crimpability. In fact, the density of the former point is $0.904\left(\mathrm{~g} / \mathrm{cm}^{2}\right)$ and that of the latter point is $0.905\left(\mathrm{~g} / \mathrm{cm}^{3}\right)$. Both agree fairly well.

\subsection{Effects of some other spinning conditions}

3.3.1 Effect of linear density of single filaments on the optimum density

The densities of urdrawn fibers optimum to produce good crimped fibers were experimentally obtained and summarized in Fig. 8. Under the condition of a certain fixed linear density, there is only one optimum density of undrawn fibers, independent of cooling conditions and spinnerete temperature, as shown in Fig. 2. But this optimum density increases with the increase in the linear density of single filaments as shown in Fig. 8. The author can not yet present the proof to clarify this phenomenon. But it seems to be originated from the shift of the curves, depicting the relation between the drawn fiber shrinkage and the undrawn fiber density shown in Fig. 4, to the right side with the increase in the linear density of single filaments.

\subsubsection{Effect of cooling air velocity}

The air velocity in asymmetrical quenching is varied. The experimental results of Fig. 9 show that the crimpability of undrawn fibers can be intensified by the increase of cooling air velocity. This may be explained as follows: the increase of air velocity $\rightarrow$ the increase in heat transfer coefficient $\rightarrow$ the increase in Biot number $\rightarrow$ the increase in temperature gradient within a filament $\rightarrow$ the increase in

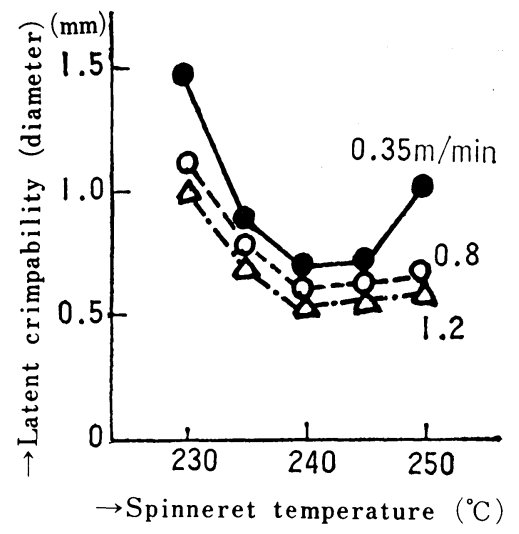

Fig. 9 The effect of quencing air velocity

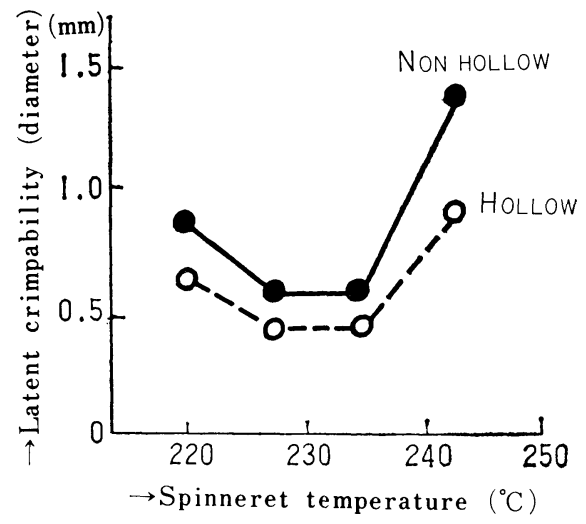

Fig. 10 The effect of hollows

crystallinity gradient within a filament $\rightarrow$ the increase in shrinkage gradient within a filament $\rightarrow$ the rise in crimpability.

\subsubsection{Effect of hollowing}

When the hollow fiber is asymmetrically quenched, the temperature difference between the windward and the leeward sides seems to be emphasized owing to the thermal insulation by air at the central part of a filament. Therefore, to make a filament hollow seems to intensify the crimpability. The experimental results shown in Fig. 10 present the proof for such an anticipation.

\section{Conclusions}

In this paper, PP crimped fibers produced by asymmetrical quenching were treated. The crimping conditions and the crimping mechanism were studied. The basic conditions to provide PP fibers with crimps are:

i) Extruded molten filaments should be asymmetrically quenched.

ii) The density of undrawn fibers thus obtained should be within a certain optimum range. 
It was clarified that the windward side of a filament has larger crystallinity and is located in the inner side of a helical crimp. Comparisons were made between experimental diameters of helical crimps and those calculated by the equation previously derived, ${ }^{[22]}$ under the assumption of certain cross-sectional shrinkage distributions. It was experimentally and theoretically shown that the effect of the linear density on the helical crimp diameter is smaller in asymmetrical quenching than in biocomponent spinning. The reason why crimpability is the largest at such an optimum density was explained by making use of drawn fiber shrinkage vs undrawn fiber density curves. Following results were also obtained:

i) The optimum undrawn fiber density increases with the increase in single filament linear density.

ii) Crimpability can be intensified with an increase of quenching air velocity or by spinning hollow fibers.

\section{Reference}

[1] M. Horio, T. Kondo; Text. Res. J., 3, 137 (1953)

[2] U.S.P. 2,542,973 (1951)
[3] U.S.P. 3,275,720 (1966)

[4] U.S.P. 3,271,943 (1966)

[5] U.S.P. 3,061,874 (1962)

[6] U.S.P. 2,730,758 (1956)

[7] U.S.P. 3,213,171 (1965)

[8] U.S.P. 3,366,722 (1968)

[9] J.P. 33-2310 (1958)

[10] J.P. 41-21572 (1966)

[11] J.P. 43-13351 (1967)

[12] J.P. 44-913 (1969)

[13] J.P. 44-10009 (1969)

[14] J.P. 43-12621 (1968)

[15] J.P. 42-13741 (1967)

[16] B.P. 1,137,027 (1968)

[17] J.P. 36-18366 (1957)

[18] J.P. 41-6210 (1966)

[19] J.P. 44-15125 (1969)

[20] U.S.P. 3,262,247 (1966)

[21] T. Matsuo, S. Kase; J. Soc. Fib. Sci. Tech., Japan, 24, 512 (1968)

[22] H. Yasuda, K. Nagano, T. Matsuo; J. Soc. Fib. Sci. Tech. Japan, 27, 168 (1971) 